\title{
Interaction of Negative Ions with the Surface of Inert Liquids
}

\author{
A. M. Dyugaev ${ }^{a, b}$, P. D. Grigor'ev ${ }^{a, b}$, and E. V. Lebedeva ${ }^{c}$ \\ ${ }^{a}$ Landau Institute of Theoretical Physics, Russian Academy of Sciences, Chenogolovka, Moscow region, 142432 Russia \\ ${ }^{b}$ Max Planck Institut für Physik komplexer Systeme, D-01187 Dresden, Germany \\ ${ }^{c}$ Institute of Solid State Physics, Russian Academy of Sciences, Chenogolovka, Moscow region, 142432 Russia \\ e-mail: lebedeva@issp.ac.ru \\ Received December 9, 2008; in final form, January 11, 2009
}

\begin{abstract}
The interaction potential of negative ions (electron bubbles) with the surface of liquid ${ }^{4} \mathrm{He},{ }^{3} \mathrm{He}$, and $\mathrm{Ne}$ has been found. In addition to the electrostatic repulsion, the contribution of the long-range Van der Waals attraction of the electron bubble to the liquid surface has been also taken into account. Competition of these repulsion and attraction forces results in the formation of a potential barrier that prevents the motion of a negative ion from the liquid to the vacuum. The temperature and electric-field dependences of the lifetime of the bubble have been determined. The theory has been compared with the experiments with negative ions in liquid ${ }^{4} \mathrm{He}$. In contrast to the conventional idea based on the hypothesis of the quantum tunneling of an electron from a bubble to a vacuum, our theory is based on the Kramers' diffusion model of the classical escape of the bubble over the potential barrier. In this model, a low-dynamic-friction approximation is applicable to liquid ${ }^{4} \mathrm{He}$ owing to a high mobility of negative ions in the superfluid.
\end{abstract}

PACS numbers: 67.55.Ig

DOI: $10.1134 / \mathrm{S} 0021364009030102$

Positively- and negatively-charged complexes placed in liquid helium are known to have fundamentally different structures. A positively-charged complex is a $\mathrm{He}^{+}$ion surrounded by polarized atoms of solid helium [1]. An electron placed in the liquid is repelled by helium atoms and forms a bubble around itself [2]. The transport properties of positively- and negativelycharged complexes are determined by their interaction with the elementary excitations of the liquid. Experiments of various types revealed a striking difference between positive ions and electron bubbles. It turned out that $\mathrm{He}^{+}$ions cannot penetrate the surface of liquid helium, whereas electrons quite easily pass through the surface into the vacuum at not too low temperature (above $1.2 \mathrm{~K}$ ).

In experiments [3, 4], the electrons were attracted to the liquid-vapor interface by the electric field $E$. It was found that the electric current associated with the electron motion through the surface decreases exponentially with temperature. The potential barrier was 30$40 \mathrm{~K}$, depending on the magnitude of the electric field.

The interaction of a point charge with a dielectric half-space is commonly approximated by the expression

$$
V(z)=A / z+e E z
$$

where

$$
A=(\varepsilon-1) e^{2} / 4 \varepsilon(\varepsilon+1) \text {. }
$$

At low temperatures, the vapor density is low and parameter $A$ is determined by the dielectric constant $\varepsilon$ of the liquid. This approximation yields an infinitely high barrier. However, it does not take into account the finite size of the charge and assumes an abrupt change in the medium properties at the liquid-vapor interface. In reality, the density of the liquid changes smoothly in a 10-Å layer. Therefore, Bruschi et al. [5] and HsingMei Huang et al. [6] attributed the finite barrier height to a cutoff of potential (1) at a distance of a few amgstrom. In early works on the electron transport from liquid helium to a vacuum $[7,8]$, the potential of shape (1) was used to determine the equilibrium Boltzmann distribution of bubbles in the liquid and the barrier height was obtained by fitting the experimental data. In [9], the shape of the potential barrier was numerically calculated $a b$ initio taking into account the pair interaction of He atoms. Although the calculation agrees well with the measurements $[3,4]$ concerning the barrier height in zero field, it does not yield a pictorial presentation of the interactions in the system that may lower the potential barrier. The theories $[7,8]$ also do not provide such a picture.

Two different models were proposed to calculate the lifetime $\tau$ of a charged bubble and to compare it with experiment. According to a diffusion model, the charged complexes that move in the electric field and reach the helium surface "burst" and the electron appears in the vacuum. Phenomenological calculation 

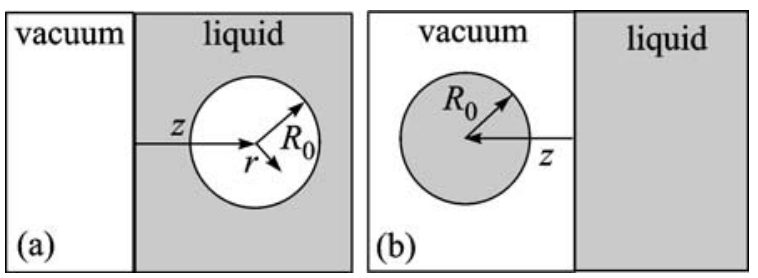

Fig. 1. Illustration of the symmetry of the Van der Waals interaction. The attraction force of the bubble to the vacuum is exactly equal to the attraction force of the cluster to the liquid surface.

[4] of the probability of such a process based on the Smoluchowski theory $[10,11]$ yields

$$
\frac{1}{\tau}=\frac{\omega_{0} \omega_{\mathrm{M}} \tau_{0}}{2 \pi} \exp \left[-\frac{V_{\mathrm{M}}}{T}+\frac{V_{0}}{T}\right] \text {. }
$$

Here, parameters $V_{\mathrm{M}}$ and $V_{0}$ are related to the expansions of the potential $V(z)$ near points $z_{\mathrm{M}}$ and $z_{0}$, respectively, where it has the maximum $V_{\mathrm{M}}$ and minimum $V_{0}$ value $[10,11]$ :

$$
\begin{gathered}
V(z)=V_{\mathrm{M}}-\frac{\left|V_{\mathrm{M}}^{\prime \prime}\right|}{2}\left(z-z_{\mathrm{M}}\right)^{2}, \\
V(z)=V_{0}+\frac{V_{0}^{\prime \prime}}{2}\left(z-z_{0}\right)^{2} .
\end{gathered}
$$

The relaxation time $\tau_{0}$ and the frequencies $\omega_{\mathrm{M}}$ and $\omega_{0}$ in Eq. (2) are determined by the mobility $\mu$ and effective dynamic mass $M$ of the negative ion [10]

$$
\tau_{0}=\frac{\mu M}{e}, \quad \omega_{0}^{2}=\frac{V_{0}^{\prime \prime}}{M}, \quad \omega_{\mathrm{M}}^{2}=\frac{\left|V_{\mathrm{M}}^{\prime \prime}\right|}{M} .
$$

The comparison of Eq. (2) with the experimental data for liquid ${ }^{4} \mathrm{He}$ performed in [4] yielded $V_{\mathrm{M}}=43.8 \mathrm{~K}$ and $\left|V_{\mathrm{M}}^{\prime \prime}\right|=2.23 \times 10^{3} \mathrm{~K} / \AA^{2}$. The parameters $V_{0}, V_{0}^{\prime \prime}$, and $z_{0}$ were found from Eq. (1):

$$
V_{0}=2 \sqrt{A e E} ; \quad V_{0}^{\prime \prime}=\frac{2 A}{z_{0}^{3}} ; \quad z_{0}^{2}=\frac{A}{e E} .
$$

Temperature dependence (2) agrees well with the experimental data but the fitting parameter $V_{\mathrm{M}}^{\prime \prime}$ appears to be three orders of magnitude larger than the reasonable estimates $[7,8]$.

This caused the rejection of the classical diffusion model for the description of negative ions in liquid ${ }^{4} \mathrm{He}$. Another model studied in detail in [7-9] suggests that the electric current across the liquid surface is due to the quantum tunneling of electrons from bubbles to a vacuum. To come to an agreement between the tunneling model and the experimental data, the authors of [7, 8] had to take a bubble radius of 25-26 A, which is 1.5 times larger than the value obtained from mobility measurements. The substitution of the conventional value
$R=17 \AA$ into the corresponding formulas yields the lifetime $\tau$ three orders of magnitude larger than the experimental value. A computer calculation of the lifetime of a bubble, taking into account its finite size [9], also differs from experiment.

Thus, the existing theory of electron bubbles near the surface of liquid helium does not give a satisfactory explanation of the experimental data.

We propose a new description of the interaction of an electron bubble with liquid helium. We first introduce the fundamental attraction of the bubble to the liquid surface associated with the long-range attraction between atoms

$$
V\left(\mathbf{r}_{1}-\mathbf{r}_{2}\right)=-\frac{\beta}{\left|\mathbf{r}_{1}-\mathbf{r}_{2}\right|^{6}} .
$$

Second, we take into account the finite size of the bubble. For that, we write the electron wavefunction in the form (see Fig. 1)

$$
\psi_{e}^{2}(\mathbf{r})=\frac{1}{4 \pi} \frac{2}{R_{0}} \frac{\sin ^{2}\left(\pi r / R_{0}\right)}{r^{2}},
$$

where $R_{0}$ is the characteristic size of the bubble. Approximation (7) implies an absolute impossibility of the quantum tunneling of the electron from the bubble to the liquid. Defining the dimensionless parameter $x=$ $z / R_{0}$, we replace the $A / z$ term in Eq. (1) by $V_{e}$ given by the formula

$$
\begin{gathered}
V_{e}=\varepsilon_{e} f_{e} ; \quad \varepsilon_{e}=\frac{A}{R_{0}} ; \\
f_{e}(x)=\int_{0}^{1} \frac{d y}{y} \ln \left(\frac{x+y}{x-y}\right) \sin ^{2} \pi y .
\end{gathered}
$$

The expression for $f_{e}(x)$ is obtained by integrating the interaction potential of the electron with the liquid surface (see Fig. 1).

$$
V_{e}(z)=A \int \frac{\psi_{e}^{2}(\mathbf{r}) d \mathbf{r}}{z(\mathbf{r})} ; \quad z(\mathbf{r})=z+r_{z} .
$$

The function $f_{e}(x)$ is defined at $x>1\left(z=R_{0}\right)$ and may be expressed as a fast-converging power in series of $1 / x$

$$
\begin{gathered}
f_{e}(x)=\frac{1}{x}\left(1+\frac{c_{2}}{x^{2}}+\frac{c_{4}}{x^{4}}\right), \\
c_{2}=\frac{1}{9}-\frac{1}{6 \pi^{2}}=0.0942, \\
c_{4}=\frac{1}{25}+\frac{3}{10 \pi^{4}}-\frac{1}{5 \pi^{2}}=0.0228 .
\end{gathered}
$$

The value $f_{e}(1)=1.13$ gives an idea of the accuracy of expansion (10). For $z=R_{0}$, the approximation of Eq. (1) by $V_{e}=A / R_{0} x$ is accurate to 0.13 . 
A more accurate computation of the function $\psi_{e}(r)$ is pointless, as even the rough approximation $\sin ^{2} \pi y=$ $1 / 2$ does not significantly change the result in Eq. (8):

$$
f_{e}(x)=\frac{1}{x}+\frac{1}{9 x^{3}}+\frac{1}{25 x^{5}} \ldots
$$

In particular, $f_{e}(1)=\pi^{2} / 8=1.23$. Thus, the inclusion of the finite size of the bubble yields only a minor numerical correction. However, we shall take it into account in our calculations.

The effects of the bubble attraction to the surface (and the repulsion of the $\mathrm{He}^{+}$ion from the surface) may be understood comparing Figs. 1a and 1b. They reflect the symmetry of matter and a vacuum: the attraction of a ball to a liquid is exactly equal to the attraction of a bubble to a vacuum. For the same reason, vacancies (holes) are concentrated near the surface of a solid. An atomic vacancy is attracted to the surface by the potential $v(z)$ obtained by integrating interaction (6) over the vacuum $(z<0)$ half-space (see Fig. 1)

$$
v(z)=-\frac{\pi}{6} \beta n \frac{1}{z^{3}} .
$$

If $z \gg R_{0}$, the bubble is attracted to the vacuum by the potential $v^{+}=v(z) N$, where $N$ is the number of particles in the bubble. Since $N \gg 1$ (see table),

$$
N \approx \frac{4}{3} \pi R_{0}^{3} n,
$$

where $n$ is the liquid density. Integrating Eq. (11) over the bubble volume, taking into account its finite size, we obtain the attraction potential of a negative ion to the liquid surface

$$
\begin{gathered}
V_{b}(x)=-\varepsilon_{b} f_{b}, \quad \varepsilon_{b}=\frac{\pi^{2} \beta n^{2}}{6} \\
f_{b}(x)=\frac{2 x}{x^{2}-1}-\ln \frac{x+1}{x-1} ; \quad x=\frac{z}{R_{0}}>1 .
\end{gathered}
$$

For $x \gg 1$,

$$
f_{b}(x) \approx \frac{4}{3} \frac{1}{x^{3}} .
$$

Thus, the total interaction potential of the bubble and the liquid surface reads as

$$
V^{-}(x)=\varepsilon_{e} f_{e}(x)-\varepsilon_{b} f_{b}(x)+e E z .
$$

We performed a numerical computation of potential (15) for ${ }^{4} \mathrm{He},{ }^{3} \mathrm{He}$, and $\mathrm{Ne}$. The results are shown in Fig. 2. The dashed lines are the respective curves obtained according to Eq. (1). Figure 3 presents the $V(z)$ curves for ${ }^{4} \mathrm{He}$ and several values of electric field $E$. The height $V_{\mathrm{M}}=39 \mathrm{~K}$ of the potential barrier for ${ }^{4} \mathrm{He}$ agrees well with the experiments [3,4]. Calculation using Eq. (15) gives a reasonable value of $\left|V_{\mathrm{M}}^{\prime \prime}\right|=0.6 \mathrm{~K} / \AA^{2}$.

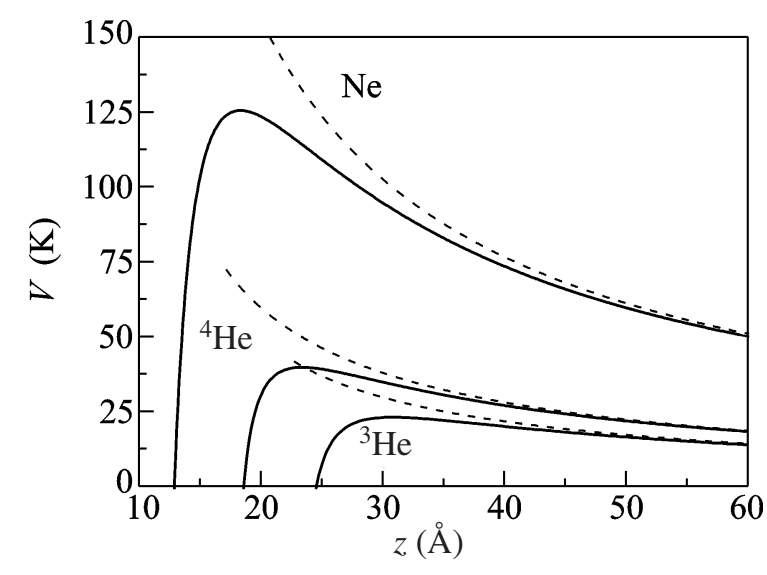

Fig. 2. Behavior of the potential $V(z)$ determined by Eq. (15) near its maximum $V_{\mathrm{M}}$ at zero electric field for three inert liquids. The dashed lines are the respective curves given by Eq. (1).

The constants used in our computation are listed in the table.

Our approach to the description of the behavior of charged complexes near the surface of a dielectric liquid provides a simple explanation of the difference between positively-charged clusters and negativelycharged bubbles. At a distance of about tens of angstroms, the interaction of the bubble and the liquid surface is a result of the competition of attraction and repulsion forces (15), whereas for the clusters, both forces are directed into the liquid.

Next, having determined the shape (15) of the potential barrier, we can calculate the lifetime of the electron bubble in the diffusion model. It is important that liquid ${ }^{4} \mathrm{He}$ is a superfluid in the temperature range $T=1.1-1.9 \mathrm{~K}$, for which the experimental results $[3,4$, 7] were obtained. Thus, the mobility of electron bubbles in this temperature range is very high. According to $[10,11]$, Eq. (2) holds only in the limiting case of a

Table

\begin{tabular}{l|l|c|c|c}
\hline & & ${ }^{4} \mathrm{He}$ & ${ }^{3} \mathrm{He}$ & $\begin{array}{c}\mathrm{Ne} \\
(T=25 \mathrm{~K})\end{array}$ \\
\hline$\varepsilon$ & & 1.057 & 1.043 & 1.19 \\
$n$ & $1 / \AA^{3}$ & 0.02184 & 0.01635 & 0.037 \\
$R_{0}$ & $\AA$ & 17.0 & 22.5 & 10 \\
$\beta$ & $\mathrm{K} \AA^{6}$ & $1.038 \times 10^{4}$ & $1.038 \times 10^{4}$ & $6.14 \times 10^{4}$ \\
$\varepsilon_{e}$ & $\mathrm{~K}$ & 63.8 & 37.1 & 304.3 \\
$N$ & & 449 & 780 & 155 \\
$\varepsilon_{b}$ & $\mathrm{~K}$ & 8.14 & 4.56 & 138.5 \\
$V_{\mathrm{M}}$ & $\mathrm{K}$ & 39 & 23 & 125 \\
$\left|V_{\mathrm{M}}^{\prime \prime}\right|$ & $\mathrm{K} / \AA^{2}$ & 0.6 & 0.21 & 1.82 \\
$\omega_{\mathrm{M}}$ & $1 / \mathrm{s}$ & $2.36 \times 10^{10}$ & $1.22 \times 10^{10}$ & $3.12 \times 10^{10}$ \\
\hline
\end{tabular}




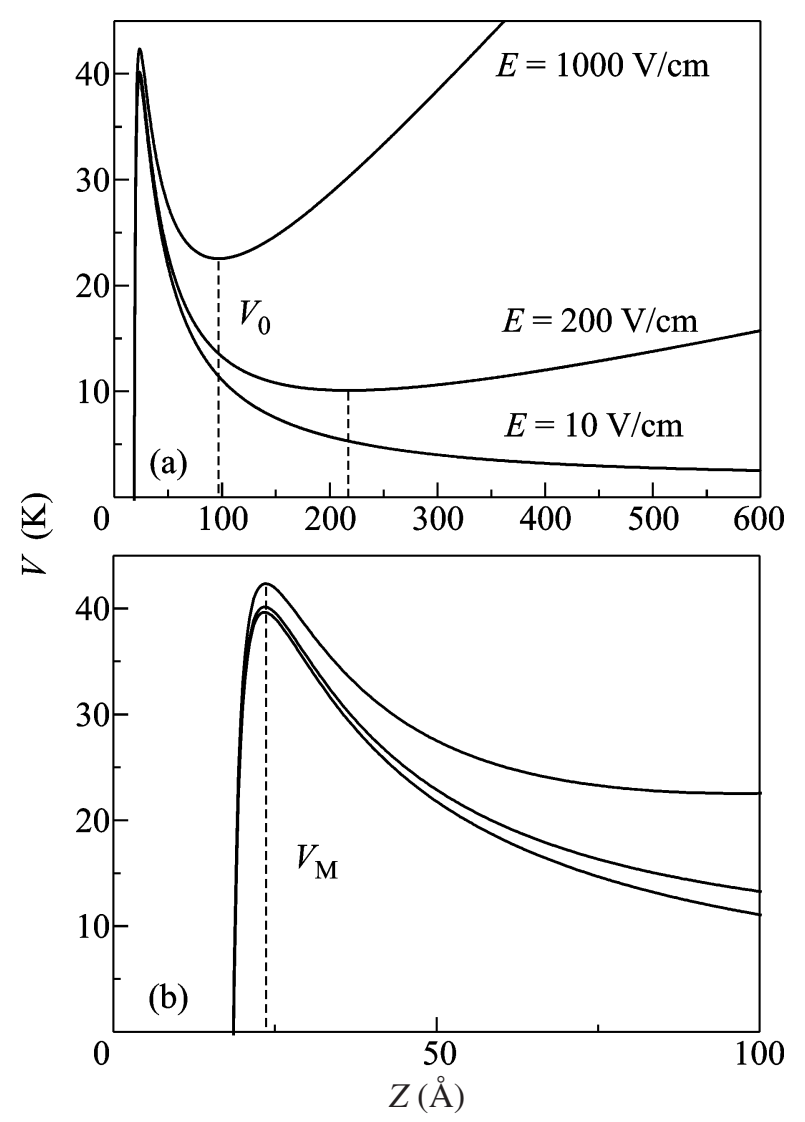

Fig. 3. (a) Potential $V(z)$ for ${ }^{4} \mathrm{He}$ at different values of the electric field $E$. (b) Behavior of $V(z)$ near its maximum. The $V_{\mathrm{M}}$ value weakly depends on $E$.

large dynamic friction coefficient. This condition is equivalent to the requirement $2 \omega_{\mathrm{M}} \tau_{0}<1$, where $\omega_{\mathrm{M}}$ and $\tau_{0}$ are given by Eqs. (4). The opposite limiting case of low dynamic friction $2 \omega_{\mathrm{M}} \tau_{0}>1$ takes place for helium. Then, the lifetime of the bubble is independent of $\omega_{\mathrm{M}}$ and $\tau_{0}$ and may be calculated from the expression $[10,11]$

$$
\frac{1}{\tau}=\frac{\omega_{0}}{2 \pi} \exp \left[-\frac{V_{\mathrm{M}}}{T}+\frac{V_{0}}{T}\right]
$$

Simple formula (16) follows from the exact solution for $\tau$ obtained by Kramers [11] at $V_{\mathrm{M}} / T \gg 1$

$$
\begin{aligned}
& \frac{1}{\tau}=\frac{\omega_{0}}{2 \pi} {\left[\left(1+\frac{1}{4 \tau_{0}^{2} \omega_{\mathrm{M}}^{2}}\right)^{1 / 2}-\frac{1}{2 \omega_{\mathrm{M}} \tau_{0}}\right] } \\
& \times \exp \left[-\frac{V_{\mathrm{M}}}{T}+\frac{V_{0}}{T}\right] .
\end{aligned}
$$

At $\omega_{\mathrm{M}} \tau_{0}<1$, Eq. (17) reduces to Eq. (2) obtained from the Smoluchowski equation. However, at $\omega_{\mathrm{M}} \tau_{0}>1$, Eq. (17) reduces to Eq. (16) corresponding to the limiting case of low dynamic friction.

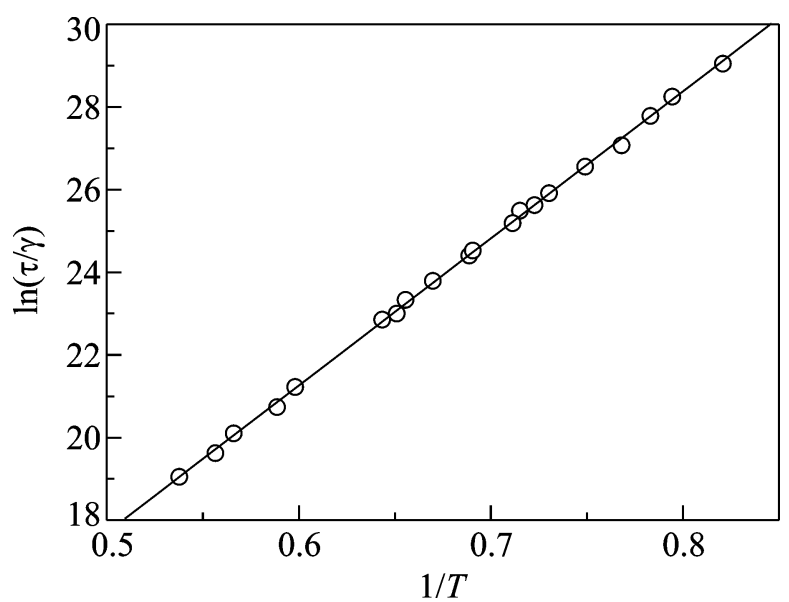

Fig. 4. Temperature dependence of the lifetime of the electron bubble. The points are the experimental data [7]. The straight line corresponds to Eq. (16) with $V_{\mathrm{M}}=35.6 \mathrm{~K}, V_{0}=$ $0.63 \sqrt{E} \mathrm{~K}$, and $\omega_{0}=18 \times 10^{6} E^{3 / 4} 1 / \mathrm{s}$. The parameter $\gamma$ is given by Eq. (19).

The mobility $\mu_{4}$ of negative ions in liquid ${ }^{4} \mathrm{He}$ in the temperature range studied in $[4,7]$ is determined by their interaction with rotons and depends exponentially on $T$ [12]:

$$
\mu_{4}(T) \approx 1.2 \times 10^{-3} e^{\Delta / T} \mathrm{~cm} / \mathrm{V} \mathrm{s}, \quad \Delta \cong 8.1 \mathrm{~K} .
$$

The $\Delta$ value in Eq. (18) is close to the roton gap width $\Delta=8.65 \mathrm{~K}$ determined from neutron scattering. The comparison of Eqs. (2) and (16) taking into account Eq. (18) explains the good agreement of the exponential dependence $\tau(T)$ obtained in [4] on the basis of Eq. (2) with the experimental data.

The comparison of Eq. (16) with the experiments [7] is presented in Fig. 4, where $\ln (\tau / \gamma)$ for ${ }^{4} \mathrm{He}$ is plotted against $1 / T$. The parameter $\gamma$ is given by the expression

$$
\gamma=\frac{2 \pi}{\omega_{0}} e^{-V_{0} / T} ; \quad \tau / \gamma=e^{V_{\mathrm{M}} / T} .
$$

The $V_{\mathrm{M}}, V_{0}$, and $\omega_{0}$ values for ${ }^{4} \mathrm{He}$ calculated from Eqs. (3), (5), and (15) are

$$
\begin{gathered}
V_{\mathrm{M}}=39 \mathrm{~K} ; \quad V_{0}=0.7 \sqrt{E} \mathrm{~K} ; \\
\omega_{0}=8 \times 10^{6} E^{3 / 4} 1 / \mathrm{s},
\end{gathered}
$$

where $E$ is expressed in $\mathrm{V} / \mathrm{cm}$. The idea of the accuracy of the low dynamic friction approximation specified by Eq. (16) for ${ }^{4} \mathrm{He}$ is given by the relation between the parameter $2 \omega_{\mathrm{M}} \tau_{0}$ and $\mu_{4}$, which comes from Eqs. (3), (4), and (18)

$$
\begin{gathered}
2 \omega_{\mathrm{M}} \tau_{0}=44 \mu_{4}(T) ; \\
\tau_{0}=10^{-9} \times 0.93 \mu_{4} \mathrm{~s} ; \quad \omega_{\mathrm{M}}=2.36 \times 10^{10} 1 / \mathrm{s} ;
\end{gathered}
$$


where $\mu_{4}$ is expressed in $\mathrm{cm}^{2} / \mathrm{V}$ s. The condition $2 \omega_{\mathrm{M}} \tau_{0}>1$ was indeed satisfied in the entire temperature range where the dependence $\tau(T)$ was measured for ${ }^{4} \mathrm{He}$ [7]. Consequently, even the simplest diffusion model applied to the electron bubble in liquid ${ }^{4} \mathrm{He}$ with the inclusion of our knowledge of the structure of the potential barrier quantitatively agrees with the experimental data. Under a change in $\tau / \gamma$ by six orders of magnitude, exponential temperature dependence (19) holds (see Fig. 4) with the values

$$
\begin{gathered}
V_{\mathrm{M}}=35.6 \mathrm{~K} ; \quad V_{0}=0.63 \sqrt{E} \mathrm{~K} ; \\
\omega_{0}=18 \times 10^{6} E^{3 / 4} 1 / \mathrm{s},
\end{gathered}
$$

which are close to the calculated values given in Eqs. (20).

To conclude, we have determined ab initio the interaction potential of negative ions and the surface of liquid ${ }^{4} \mathrm{He}(15)$ and calculated the lifetime of the electron bubbles from Eq. (16). In contrast to the theory of the quantum tunneling of electrons from bubbles to a vacuum [7-9], our results are based on the classical diffusion model $[10,11]$. We finally note that the proposed theory of surface phenomena associated with negative ions is also applicable to ${ }^{3} \mathrm{He},{ }^{3} \mathrm{He}-{ }^{4} \mathrm{He}$ solutions, and Ne. However, exact formula (17) derived by Kramers in 1940 [11] should be applied to these inert liquids.
This work was supported the Russian Foundation for Basic Research, project nos. 06-02-16551 and 06-02-16223.

\section{REFERENCES}

1. K. R. Atkins, Phys. Rev. 116, 1339 (1959).

2. R. A. Ferrel, Phys. Rev. 108, 167 (1957).

3. L. Bruschi, B. Maraviglia, and F. E. Moss, Phys. Rev. Lett. 17, 682 (1966).

4. W. Schoepe and C. Probst, Phys. Lett. A 31, 490 (1970).

5. L. Bruschi, G. Mazzi, M. Santini, and G. Torzo, J. Phys. C: Solid State Phys. 8, 1412 (1975).

6. Hsing-Mei Huang, Yu Ming Shih, and Chia-Wei Woo, J. Low Temp. Phys. 14, 413 (1974).

7. W. Schoepe and G. W. Rayfield, Phys. Rev. A 7, 2111 (1973).

8. Milton W. Cole and James R. Klein, J. Low Temp. Phys. 36, 331 (1979).

9. Francenso Ancilotto and Flavio Toigo, Phys. Rev. B 50, 12820 (1994).

10. S. Chandrasekhar, Rev. Mod. Phys. 15, 63 (1943).

11. H. A. Kramers, Physica 7, 284 (1940).

12. F. Reif and L. Meyer, Phys. Rev. 119, 1164 (1960).

Translated by A. Safonov 